\title{
Improving adaptive subtraction in seismic multiple attenuation
}

\author{
Shoudong Huo ${ }^{1}$ and Yanghua Wang ${ }^{2}$
}

\begin{abstract}
In seismic multiple attenuation, once the multiple models have been built, the effectiveness of the processing depends on the subtraction step. Usually the primary energy is partially attenuated during the adaptive subtraction if an $\mathrm{L}^{2}$-norm matching filter is used to solve a least-squares problem. The expanded multichannel matching (EMCM) filter generally is effective, but conservative parameters adopted to preserve the primary could lead to some remaining multiples. We have managed to improve the multiple attenuation result through an iterative application of the EMCM filter to accumulate the effect of subtraction. A Butterworth-type masking filter based on the multiple model can be used to preserve most of the primary energy prior to subtraction, and then subtraction can be performed on the remaining part to better suppress the multiples without affecting the primaries. Meanwhile, subtraction can be performed according to the orders of the multiples, as a single subtraction window usually covers different-order multiples with different amplitudes. Theoretical analyses, and synthetic and real seismic data set demonstrations, proved that a combination of these three strategies is effective in improving the adaptive subtraction during seismic multiple attenuation.
\end{abstract}

\section{INTRODUCTION}

A wave-equation-based multiple attenuation method usually consists of two steps, multiple prediction and adaptive subtraction (Wiggins, 1988; Verschuur et al., 1992; Berkhout and Verschuur, 1997; Verschuur and Berkhout, 1997; Weglein et al., 1997; Wang, 2004, 2007; Lu, 2006; Kaplan and Innanen, 2008), which are equally important for producing a satisfactory multiple attenuation result. Here, we focus on the adaptive subtraction. We attempt to improve the effectiveness of subtraction through iterative application of the expanded multichannel matching (EMCM) filter, masking the data prior to subtraction and subtracting multiples according to their orders.

First, an effective matching filter is the kernel of the adaptive subtraction. The expanded version of the commonly used multichannel matching filter, the EMCM filter, not only can explore effectively the lateral coherency of adjacent traces but also increase the resolution in the time axis (Wang, 2003b). Three essential parameters in the EMCM filter are the filter length, window size in time, and number of channels in matching. We find in the application that the choice of the best parameters is fundamental to an optimal multiple attenuation result, and the iterative application of the filter can improve the result further. Moreover, this iteration is performed in the subtraction step, and it is numerically cheaper than a similar iteration implementation adopted in the more expensive multiple modeling step to improve the dynamic properties of multiple models (Berkhout and Verschuur, 1997; Wang, 2004, 2007).

Second, masking the data prior to multiple suppression also is an essential factor in the process. The basic idea (Zhou and Greenhalgh, 1994, 1996; Landa et al., 1999; Kelamis et al., 2002; Wang, 2003a) is to use predicted multiples as references to design a masking filter so as to separate most of the primary energy. We apply it during adaptive subtraction in the $t-x$ domain, before adaptively subtracting modeled multiples from only the remaining part. This application has been proved to be the most efficient way to preserve the primary energy.

Third, mixed-order multiples are obstacles to a successful multiple attenuation in some cases. All orders of surface-related multiples can be modeled via spatial and temporal convolution (Verschuur et al., 1992; Berkhout and Verschuur, 1997), but multiples with different orders usually possess different properties. The multiple model, although considered to be kinematically accurate, hardly can obtain correct properties such as amplitude. Moreover, a single time window sometimes could cover different-order multiples during the adaptive subtraction. It is therefore difficult to eliminate all the multiples simultaneously because of the limitation of multiple modeling.

Manuscript received by the Editor 1 June 2008; revised manuscript received 12 January 2009; published online 19 May 2009.

${ }^{1}$ Formerly Imperial College London, Department of Earth Science and Engineering, Centre for Reservoir Geophysics, South Kensington, London, U. K.; presently Saudi Aramco, EXPEC Advanced Research Center, Geophysics Technology Team, Dhahran, Saudi Arabia. E-mail: shoudong.huo.1@aramco.com.

${ }^{2}$ Imperial College London, Department of Earth Science and Engineering, Centre for Reservoir Geophysics, South Kensington, London, U. K. E-mail: yanghua.wang@imperial.ac.uk.

(C) 2009 Society of Exploration Geophysicists. All rights reserved. 
A possible solution to this might be the adoption of a small time window, but it cannot tackle the problem completely. Hugonnet (2002) introduced a partial solution, which builds multiple models by convolving a portion of primaries (the shallow part without contamination of multiples) with the whole data set. Baumstein and Hadidi (2006) also proposed using accurate primaries to build different-order multiple models after the dip moveout (DMO) reconstruction of 3D marine data. Kaplan and Innanen (2008), on the other hand, used independent component separation strategy to eliminate the mixed-order multiples. Here, we introduce a refining procedure of surface-related multiple elimination (SRME) by modeling and subtracting separate-order multiples with previous SRME results. We demonstrate that it is much more effective to separately subtract mixed-order multiples with different amplitude magnitudes according to their orders.

\section{ITERATIVE EMCM FILTERING}

Given a raw data trace $d(t)$ and a group of $N$ multiple-model traces $m_{j}(t)$, the EMCM filter can be expressed as (Wang, 2003b)

$$
\begin{aligned}
p(t)= & d(t)-\sum_{j=1}^{N}\left[f_{1, j}(t) * m_{j}(t)+f_{2, j}(t) * \dot{m}_{j}(t)\right. \\
& \left.+f_{3, j}(t) * m_{j}^{H}(t)+f_{4, j}(t) * \dot{m}_{j}^{H}(t)\right],
\end{aligned}
$$

where $f_{i, j}(t)$ are the shaping filters, * indicates convolution, and the residual $p(t)$ is the multiple attenuation result. In equation $1, m_{j}{ }^{H}(t)$ is the Hilbert transform of $m_{j}(t) ; \dot{m}_{j}(t)$ and $\dot{m}_{j}^{H}(t)$ are the derivatives of $m_{j}(t)$ and $m_{j}{ }^{H}(t)$, respectively. Compared with the conventional multichannel matching filter, the EMCM approach expands not only the number of traces but also new physical dimensions consisting of three adjoined traces mathematically derived from the multiple model trace $m_{j}(t)$. Three parameters affect the above subtraction: filter length, number of channels, and window size in the adaptive subtraction. It is essential to find appropriate parameters that not only can remove multiple events, but also preserve the primaries at the same time.

A multichannel approach helps to suppress random noises and preserve the primaries through use of the lateral coherence of neighboring traces. However, the quality of the multiple attenuation result does not always improve with the increase in number of matching channels. In practice, the lateral coherence decreases when the involving traces exceed a certain number (Spitz, 1999), and the key lies in the choice of the correct channel number for different data sets.

The window length influences the quality of the autocorrelation and crosscorrelation that constitute the normal equation in the above least-squares problem (Treitel, 1970). A long time window behaves like the adjacent traces to serve as the vertical coherence and consequently helps to preserve the primaries. Verschuur and Berkhout (1997) suggest the adoption of long global filters to take care of the source signature. However, using long windows for local filters can have a risk of covering different-order multiples within one window, and thus affect the result of the conventional adaptive subtraction.

Compared with the above two parameters, filter length is the most important and effective parameter. The criterion of the matching filter is the minimum energy of the residual. Long filter length matches the data well and thus removes more data residual during the subtraction. Theoretically, the residual drops to zero when the filter length is increased to infinity. It is therefore difficult to preserve the primary energy contained in the residual if the filter length is too long. Furthermore, a long filter is more likely to match the multiple model to primaries when primary and multiple events are close to each other. Therefore we recommend the adoption of a filter length that is the same as or slightly longer than the source signature.

Although a short matching filter can reduce the risk of matching multiples to primaries, it cannot eliminate all the multiple energy in just one step. Most iterative methods, e.g., steepest descent and conjugate gradient approaches, gradually improve the solution. Inspired by the steepest descent method, we applied the EMCM filter iteratively to optimize the demultiple result.

To explain the approach, we use the simple single-channel filter as an example:

$$
p(t)=d(t)-f(t) * \tilde{m}(t)
$$

where $\tilde{m}(t)$ is the multiple model. The iterative approach can be defined as

$$
p^{(i)}(t)=d(t)-f^{(i)}(t) * \widetilde{m}^{(i)}(t)
$$

where $i$ is the iteration number. The multiple model is generated in the multiple prediction phase, for $i=1, \widetilde{m}^{(i)}(t)=\widetilde{m}(t)$, and for $i>1$,

$$
\tilde{m}^{(i)}(t)=d(t)-p^{(i-1)}(t) .
$$

The iteration increases the actual filter length. Take a three-iteration application, for example,

$$
p^{(3)}(t)=d(t)-f^{(3)}(t) * f^{(2)}(t) * f^{(1)}(t) * \tilde{m}(t),
$$

where the actual matching filter after the third iteration is $f^{(3)}(t) * f^{(2)}$ $(t) * f^{(1)}(t)$. If we set the filter length in each iteration as $\ell$ (samples), the final filter length will be $3 \ell-2$, much longer than the original setting. As longer matching filters eliminate more multiple energy, the remaining multiple energy can be reduced further by the iterations, so that we can select the best approximate result. This approach is numerically cheap as it implements the iteration in the subtraction step, whereas similar concepts, such as iterative SRME and multiple prediction through inversion (MPI), perform iterations in the more expensive multiple modeling step.

We have conducted many experiments on several data sets using single-channel, normal multichannel, and EMCM methods to test the effects of the parameters mentioned above. The EMCM generally is better than single-channel and normal multichannel matching methods. These experiments reveal that long window size and short filter length tend to produce a better result within the EMCM, and the iterations could further improve the result.

In the Pluto synthetic data (Figure 1a), several orders of multiples exist, and the second-order water-bottom multiples are weak in amplitude compared to the first-order multiples. The multiple model (Figure $1 \mathrm{~b}$ ) obtained by conventional spatial convolution has predicted the position of the multiples precisely, compared with the multiple energy in the original input data, which proves its kinematic correctness. However, it also is quite obvious that the energy of the second- and high-order multiples is relatively higher than that of the first-order ones in the model.

We partially zoom in three demultiple results shown in Figure $1 \mathrm{c}-\mathrm{e}$, and use white arrows to point out the differences between them 
in Figure 2a-c, respectively. The zoom-in area is located in the white frame in the middle of Figure 1a. Figures 1c and 2a show the multiple attenuation result through application of the EMCM subtraction after the first iteration, and Figures $1 \mathrm{e}$ and $2 \mathrm{c}$ show the results after three iterations. The filter parameters used in the EMCM method are: channels $=3$, window length $=3500 \mathrm{~ms}$, and filter length $=32 \mathrm{~ms}$. Figures $1 \mathrm{~d}$ and $2 \mathrm{~b}$ show a relatively long filter approach: channels $=3$, window length $=3500 \mathrm{~ms}$, and filter length $=96 \mathrm{~ms}$.

We can observe from the comparison that the long-filter and iterative approaches contribute to better attenuation of the multiple energy. As discussed before, the iteration increases the filter length effectively. In this case, the iterative approach (Figure 2c) has an equivalent filter length of $96 \mathrm{~ms}$, the same as the long-filter approach (Figure $2 b$ ), but it obtains a better attenuation result as the long filter has a)

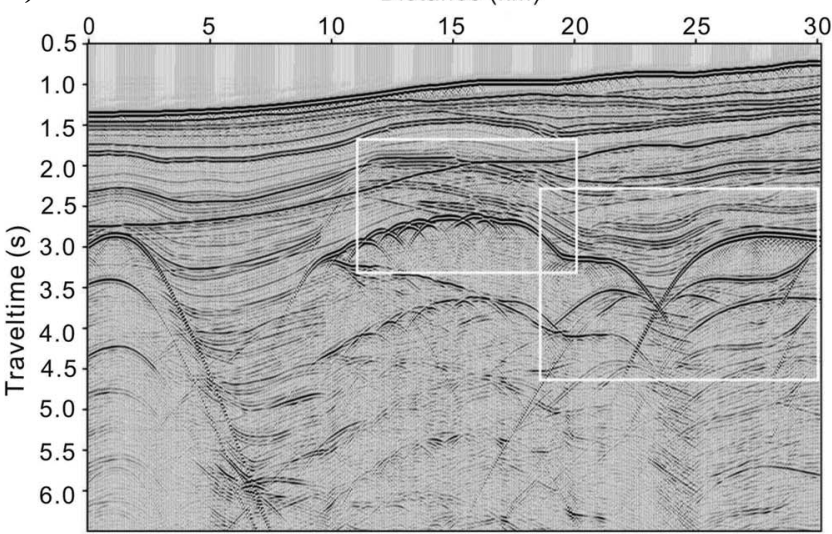

d)

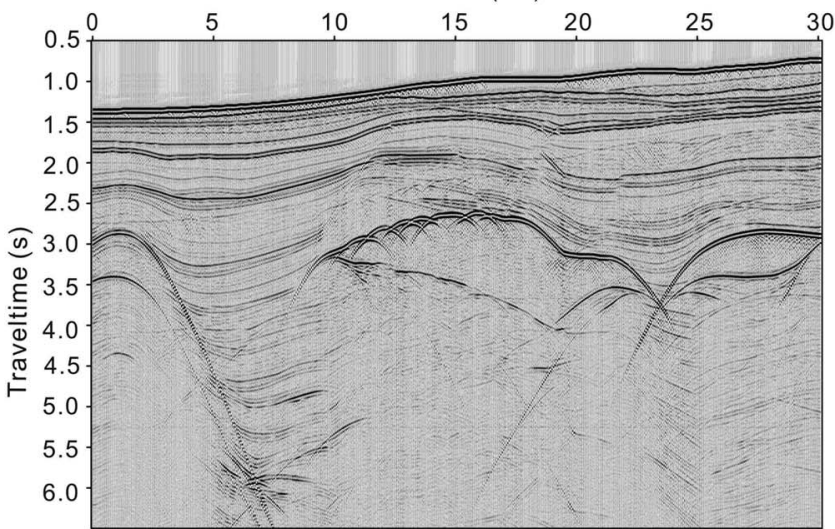

e)

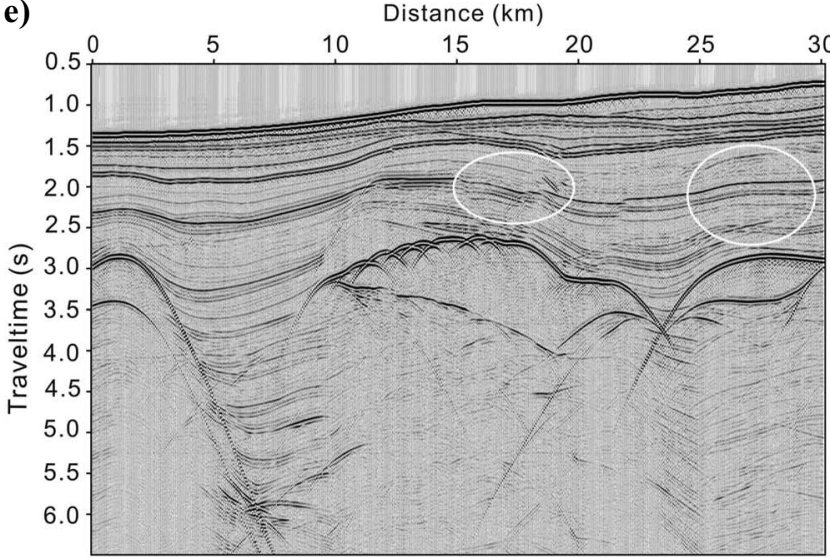

f)

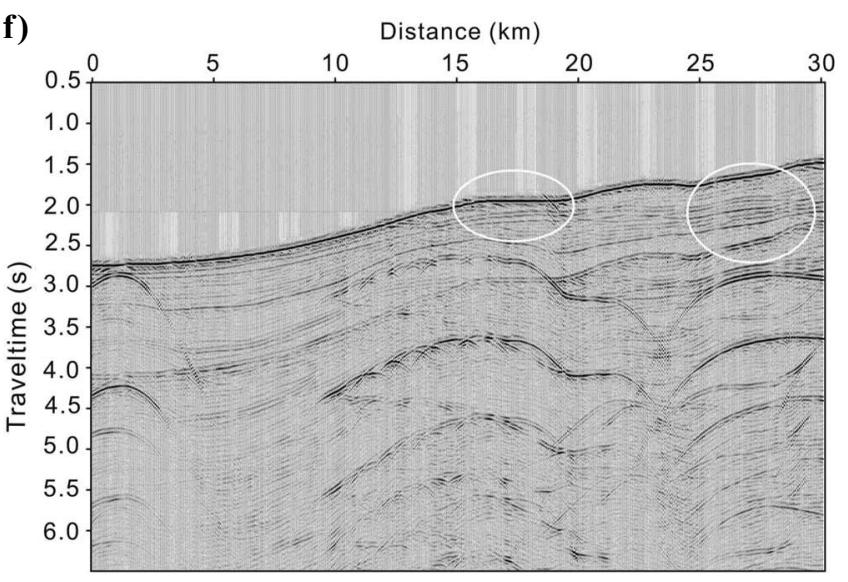

c)
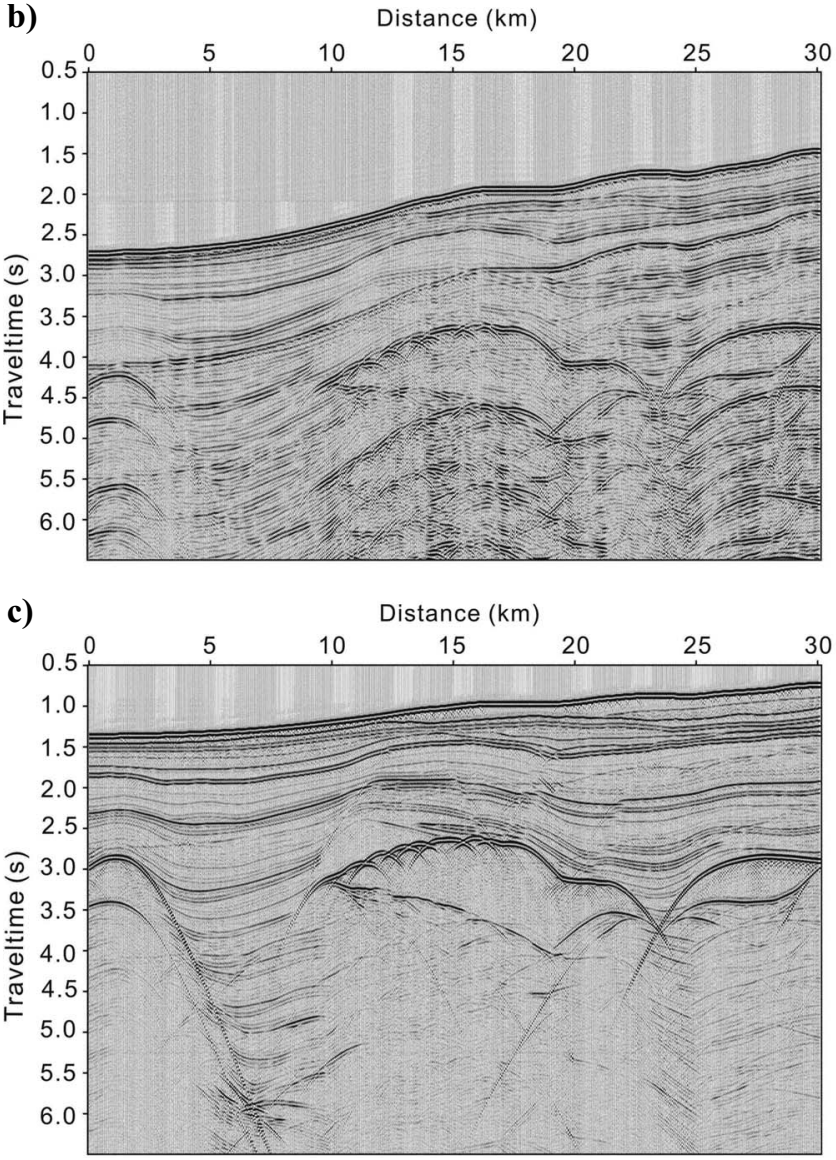

Figure 1. Application of iterative EMCM. (a) Stack section of the Pluto synthetic data. (b) Predicted multiple model with all the orders. (c) Multiple attenuation result by the EMCM method with short filter length (window $=3500 \mathrm{~ms}$, filter $=32 \mathrm{~ms}$ ) after the first iteration, and (d) with long filter length (window $=3500 \mathrm{~ms}$, filter $=96 \mathrm{~ms}$ ), (e) by using the EMCM filter three times (window $=3500 \mathrm{~ms}$, filter $=32 \mathrm{~ms}$ ). (f) Attenuated energy (i.e., the difference between [a and e]) by the iterative EMCM method. 
more chances to match the multiple model to primary events. Some artifacts (at about $1 \mathrm{~km}, 4.3 \mathrm{~s} ; 12-15 \mathrm{~km}, 2.3 \mathrm{~s}$ ) can be observed in Figures $1 \mathrm{~d}$ and $2 \mathrm{~b}$ for the above-mentioned reasons.

Figure 2 also shows the attenuated multiple energy. The multiples have been suppressed fully, but unfortunately some primary energy between 2.7 and $3 \mathrm{~s}$ is wrongly removed along with multiples. However, the iterative approach (Figure 2c) performs better than the long-filter approach (Figure 2b) as it attenuates less primary energy and produces fewer artifacts.

In the adaptive subtraction discussed above, the EMCM filter with a long window length $(3500 \mathrm{~ms})$ is applied and the two orders of water-bottom multiples are included in a single time window. Some artifacts, at about $18 \mathrm{~km}, 2.2 \mathrm{~s}$ (inside the elliptical circle), are produced along with the first-order water-bottom multiples, and the second-order ones are oversubtracted because of the low amplitude, especially between 18 and $20 \mathrm{~km}$ at $2.9 \mathrm{~s}$. Similar problems also can be observed between 26 and $28 \mathrm{~km}$ at 1.7 and $2.5 \mathrm{~s}$ (inside the elliptical circle). This leads to the following two schemes to improve further the effectiveness of multiple subtraction.

\section{MULTIPLE SUBTRACTION WITH A MASKING FILTER}

One of the fundamental assumptions in the adaptive subtraction (equation 1) is that the multiple-free primaries have the minimum energy. Thus, what we do here is use a mask on the data to protect the primary energy in the best possible way, and then perform energy minimization (i.e., multiple subtraction) on the remaining data.

The mask filter has been used in the $f-k$ domain, Radon transform domain, or the $\mathrm{t}$-x domain for multiple attenuation to reduce the leakage of primary energy (Zhou and Greenhalgh, 1994, 1996; Landa et al., 1999; Kelamis et al., 2002; Wang, 2003a). Guitton (2005) also used a masking operator in the pattern-based multiple attenuation method, which defined the operator as a diagonal matrix filled with zeros and ones, to preserve the signal when there are no multiples.

We use the nonlinear masking filter $\phi$ in an adaptive manner to preserve the primary energy before subtraction. It is adaptive in the sense that the filter is dependent on the original data and the multiple model, and is defined as a Butterworth-type function:

$$
\phi=1-\frac{1}{\sqrt{1+\left[\frac{B}{\varepsilon A}\right]^{2 n}}},
$$

where $B$ is the amplitude of the multiple model, $A$ is the amplitude of the original input section, $n$ is the parameter used to control the smoothness of the filter, and $\varepsilon$ is a weighting factor. The original data can be divided into two parts with the constraint of the masking filter: $(1-\phi) d$, the primary energy uncontaminated by multiples, and $\phi d$, the multiple energy with partly remaining primary energy. The first part of the data will not be involved in the subtraction step.
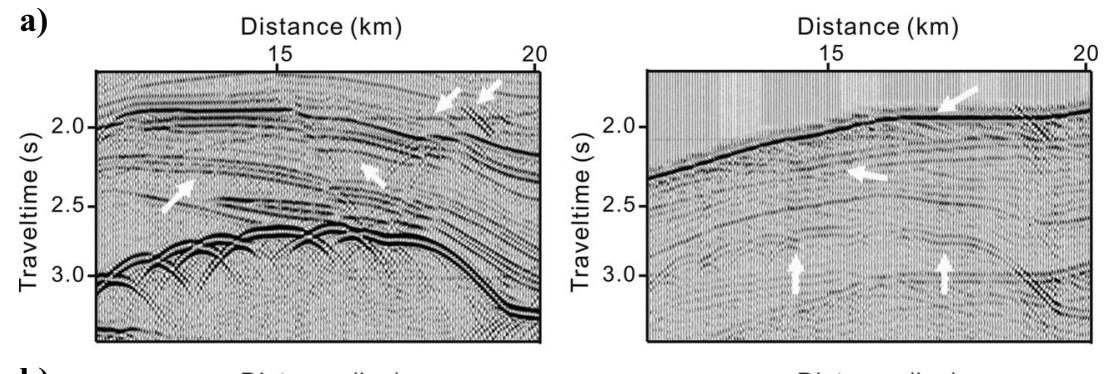

b)

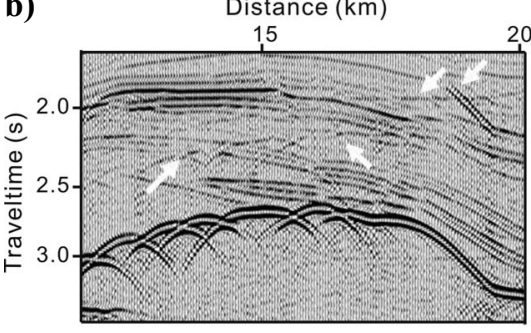

\section{政}

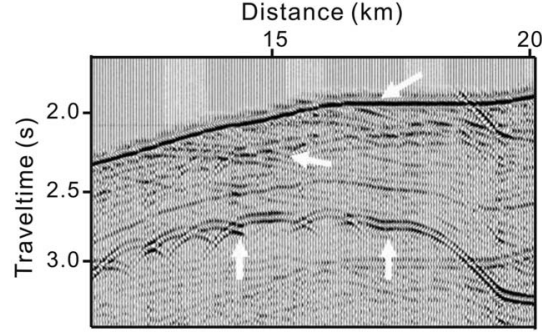

c)
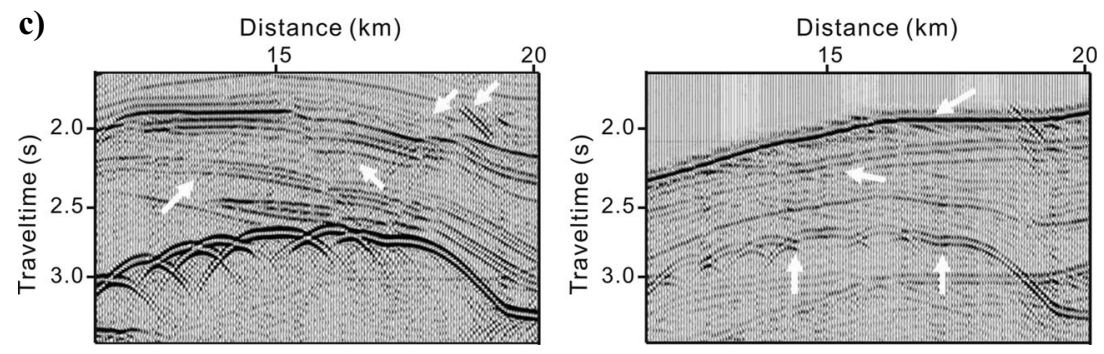

Figure 2. Partial zoom-in of application of iterative EMCM: Attenuation result (left) and attenuated energy (right). (a) Multiple attenuation result by the EMCM method with short filter length (window $=3500 \mathrm{~ms}$, filter $=32 \mathrm{~ms}$ ) after the first iteration, and $(\mathrm{b}$ ) with long filter length (window $=3500 \mathrm{~ms}$, filter $=96 \mathrm{~ms}$ ), (c) by using the EMCM filter three times (window $=3500 \mathrm{~ms}$, filter $=32 \mathrm{~ms}$ ).

\section{MULTIPLE ATTENUATION ACCORDING TO ORDERS}

It is common to cover different-order multiples in one window in the case of shallow water or long matching window, and this certainly hampers the effect of multiple subtraction. The traditional alternative is to adjust the window length of the subtraction so that it can cover only one order of multiple at a time. However, this method has two disadvantages. First, it forces us to select short window lengths when a long one is preferred during the adaptive subtraction. Second, it still cannot handle the mixed-order multiples located below the water-bottom ones as these multiples cannot be distinguished by time differences. The multiple prediction through inversion (MPI) method (Wang, 2004, 2007) can refine the dynamic properties of the model of different-order multiples and thus improve the subtraction outcome. However, the result is not always satisfactory as the method still subtracts all orders of multiples in one step.

The upgoing waves sometimes are reflected back from the surface more than once and produce high-order multiples. Multiples with the same order normally have similar amplitude and phases, and multiples with different orders might differ in properties because of the absorption and reflectivity. It is almost impossible to predict a multiple model with correct property ratios be- 
tween different-order multiple events, and the effect then is passed on to the adaptive subtraction step. For example, a matched filter $\Lambda_{i}$ can be calculated to shape the multiple model $\tilde{\mathbf{M}}_{i}$ for an event of $i$ th-order multiple $\mathbf{M}_{i}$,

$$
\mathbf{M}_{i}=\Lambda_{i} \tilde{\mathbf{M}}_{i}
$$

and similarly, another matching filter $\Lambda_{j}$ exists for an event of $j$ th-order multiple $\mathbf{M}_{j}$,

$$
\mathbf{M}_{j}=\Lambda_{j} \tilde{\mathbf{M}}_{j},
$$

where matrices in equations 7 and 8 represent data and filters in the frequency domain.

The two matching filters $\Lambda_{i}$ and $\Lambda_{j}$ are distinct from each other as the properties of $\mathbf{M}_{i}$ and $\mathbf{M}_{j}$ are normally different. Consequently, at least one of these two events cannot be well subtracted, as only one compromised filter is produced in one time window. To solve fundamentally the mixed-order multiple problem theoretically, we test an approach that produces the multiple models for each single order and then subtracts them from the original data separately.

According to the principle of SRME, prediction of different-order multiple models can be formulated as

$$
\tilde{\mathbf{M}}_{1}=\mathbf{P P}, \quad \text { and } \quad \tilde{\mathbf{M}}_{i+1}=\mathbf{P} \tilde{\mathbf{M}}_{i},
$$

where $\tilde{\mathbf{M}}_{1}$ is the first-order multiple model, $\tilde{\mathbf{M}}_{i}$ is the $i$ th-order multiple model, and $\mathbf{P}$ is the input multiple-free data that can be obtained by any multiple attenuation method. As the start of the whole procedure, it does not need to be precise because the major obstacle of mixed-order multiples during adaptive subtraction is the oversubtraction of high-order multiples. As long as most multiples are suppressed, the effect of remaining ones in $\mathbf{P}$ can be ignored during the modeling.

Theoretically, the sequence will make no difference for the subtraction, but subtracting high-order multiples first might give a slightly better result in practice. This is because high-order multiples locate at a deeper position, and subtracting them first will make less impact on low-order multiples.

Because the computation time increases linearly with the increase of the number of models we build, we recommend its use only when the conventional SRME fails. Furthermore, we simply can use $\tilde{\mathbf{M}}_{1}$ $=\mathbf{P P}$ and $\tilde{\mathbf{M}}_{h}=\mathbf{P M}$ in the adaptive subtraction when only the firstand second-order multiples are obvious in a specific data set. The attenuated multiple we get at the beginning is $\mathbf{M}$, and $\tilde{\mathbf{M}}_{h}$ is the second and high-order multiple model. This simplification makes the method efficient and easy to use, and similar strategy can be used between second- and third-order multiples, and so on.

In this approach, subtraction might be expressed as

$$
\mathbf{P}=\mathbf{D}-\Lambda\left(\tilde{\mathbf{M}}_{1}+\tilde{\mathbf{M}}_{h}\right),
$$

where $\mathbf{D}$ is the original data set. The subtraction can be implemented in sequence as

$$
\mathbf{P}^{*}=\mathbf{D}-\Lambda_{1} \tilde{\mathbf{M}}_{h}, \quad \text { and } \quad \mathbf{P}=\mathbf{P}^{*}-\Lambda_{2} \tilde{\mathbf{M}}_{1} .
$$

We use the iterative EMCM method in equation 11 to optimize the demultiple result by iterations.

\section{APPLICATIONS}

In the previous sections, we have discussed three strategies for multiple subtraction: iterative EMCM filtering, masking filters before subtraction, and subtraction according to orders. Altogether, they could improve the multiple attenuation result, as shown in Figure 3 .

A masking filter divides the original data (Figure 1a) into two parts based on the multiple model (Figure 1b). Figure $3 a$ is the part of the primary energy that must be preserved, and Figure $3 \mathrm{~b}$ is the remaining energy, which includes all the multiples. Figure $3 \mathrm{a}$ is not involved in the subtraction process at all, and Figure $3 \mathrm{~b}$ is the input data set for various subtraction experiments.

Figure $3 \mathrm{c}$ is the multiple attenuation result, in which the secondand higher-order multiples are suppressed from Figure $3 \mathrm{~b}$. The model of second- and higher-order multiples is calculated in equation 9, where matrices $\mathbf{P}$ and $\mathbf{M}_{1}$ represent shot gathers corresponding to stack sections in Figure 1d and e, respectively.

The first-order multiple model can be generated with equation 9, in which matrix $\mathbf{P}$ represents shot gathers corresponding to Figure $1 d$. Figure $3 \mathrm{~d}$ is the result after further attenuation of the first-order multiples. All orders of multiples are attenuated up through this point, and only the primary energy remains. Figure $3 \mathrm{e}$ is the final result, the sum of the preserved primary energy (Figure 3a) and demultiple result of Figure 3d. We can see that the multiple events are suppressed thoroughly without introducing any artifacts, and the continuity of primary events is maintained well (inside the elliptical circles).

Figure $3 \mathrm{f}$ shows the total attenuated energy, the difference between Figures $1 \mathrm{a}$ and $3 \mathrm{e}$. The wrongly attenuated primary events observed in Figure 1e do not appear in this figure (inside the elliptical circles), which indicates that these two schemes, together, can improve the preservation of primaries in multiple subtraction.

Figure $3 \mathrm{~g}$ indicates the result of the conventional short-window approach to handle the mixed-orders problem. We use the output of the second iteration of SRME as the multiple model because it has the similar costs, and change the window length to $800 \mathrm{~ms}$ to avoid covering different-order multiples in one window. For a better comparison, we use the same masking filter as in the previous approach. The subtraction, however, cannot preserve primaries well as the time window is not long enough to serve as a sufficient constraint. Figure $3 \mathrm{~h}$ shows the attenuated energy where strong primary events can be observed.

Figure 4 shows the zoom-in of Figure $3 \mathrm{e}-\mathrm{h}$, in which white arrows point to the events of interest for better comparison. The zoom-in areas are indicated by the white frames in Figure 1a. The arrow at the left, bottom corner, points to the multiple event in Figure $4 \mathrm{c}$ and d, and all other arrows point to the primaries that will be preserved. These figures clearly show that the subtraction according to orders does have some advantages over the iterative SRME with short window.

Figure 5a is the stack profile of a real marine seismic data set, acquired in an area with relatively deep water. It contains the free-surface multiples with three different orders covering the primaries. Figure $5 \mathrm{~b}$ is the result of conventional adaptive subtraction with masking filter. It might be observed that strong reflections between $0.9 \mathrm{~s}$ and $1.0 \mathrm{~s}$ cause some artifacts below the layers during the 
a)

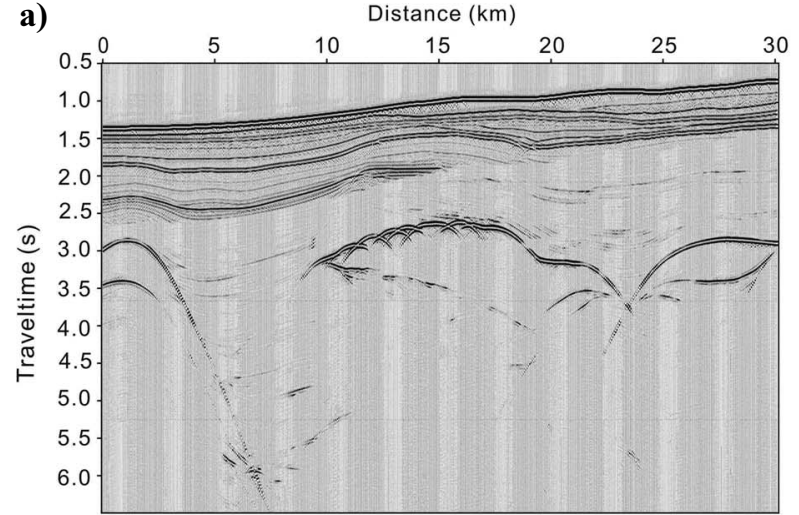

$$
\text { b) }
$$

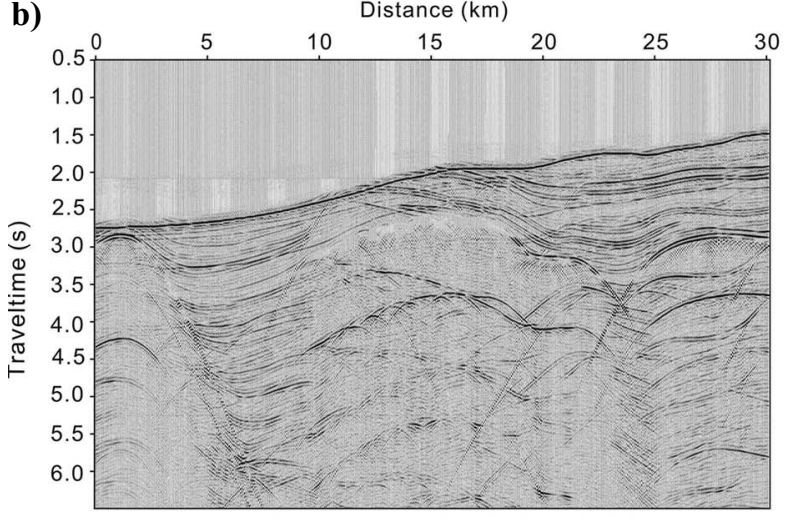

c)

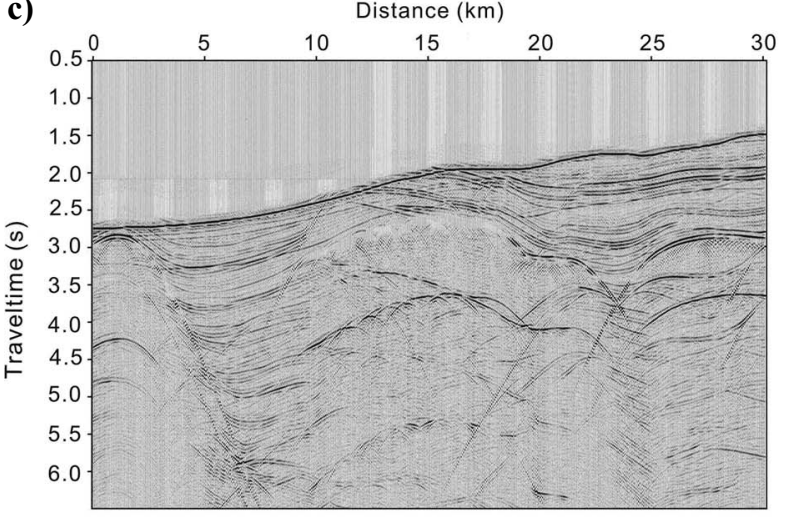

d)

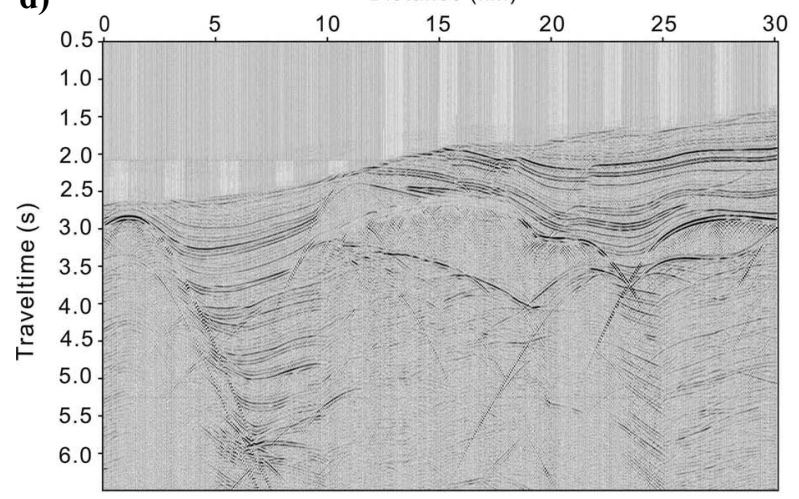

e)

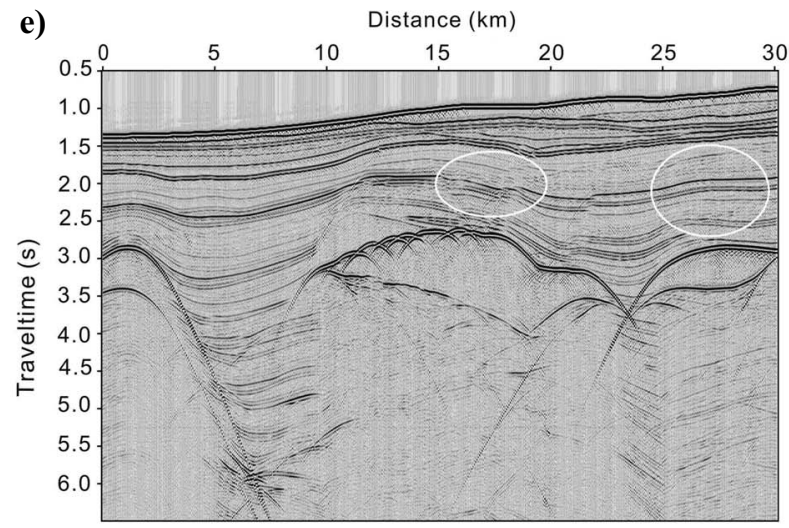

f)

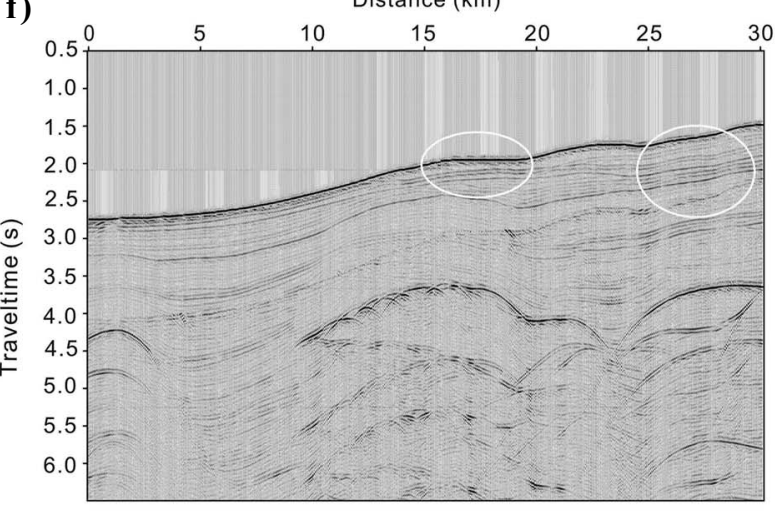

g)

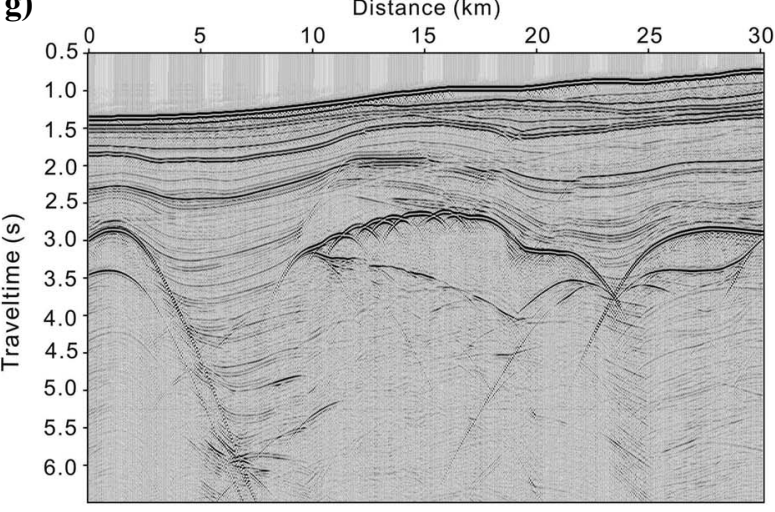

h)

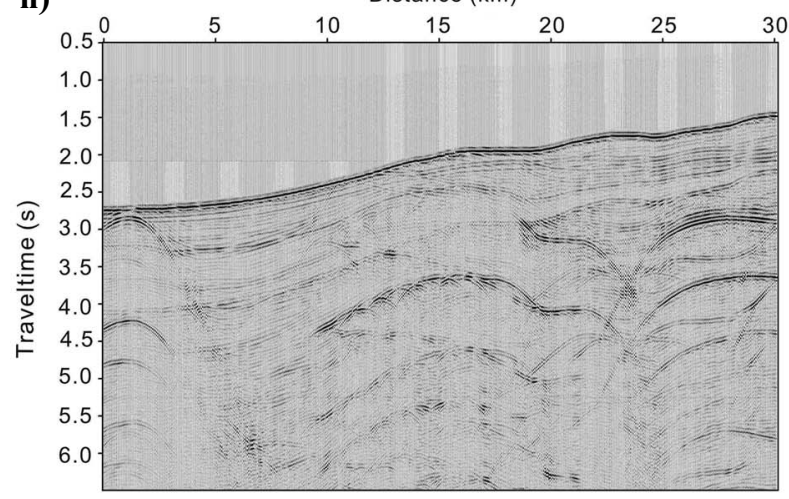

Figure 3. Application of the masking filter and subtraction according to orders. (a) Preserved primary energy. (b) The remaining primaries and multiples after masking. (c) The result after the second- and higher-order multiple attenuation. (d) The further result after the first-order multiple attenuation. (e) The final demultiple result, the combination of (a and d), and (f) the attenuated multiple energy. (g) The result of the conventional short-window (800 ms) adaptive subtraction, and (h) the attenuated multiple energy. 
a)

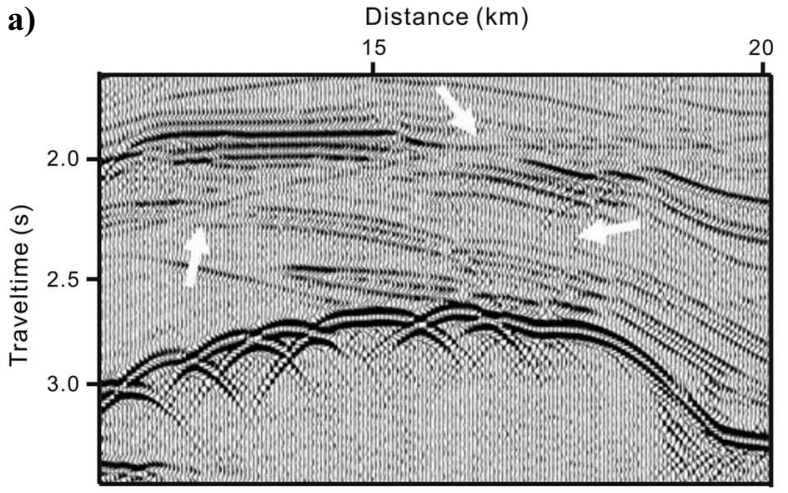

b)

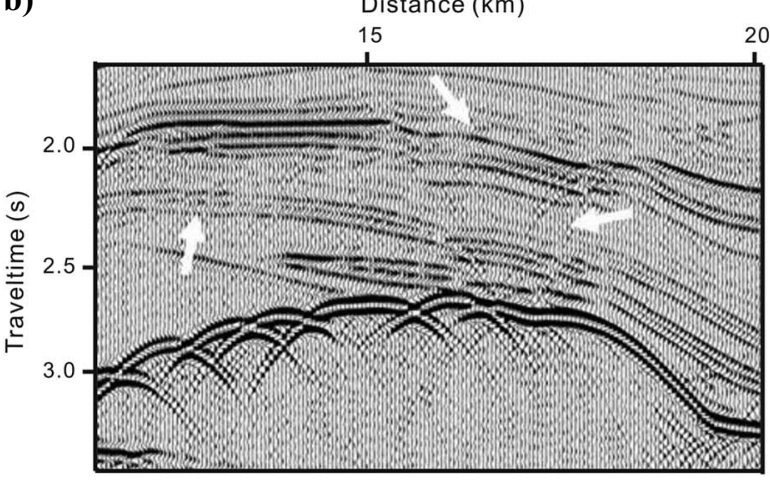

c)

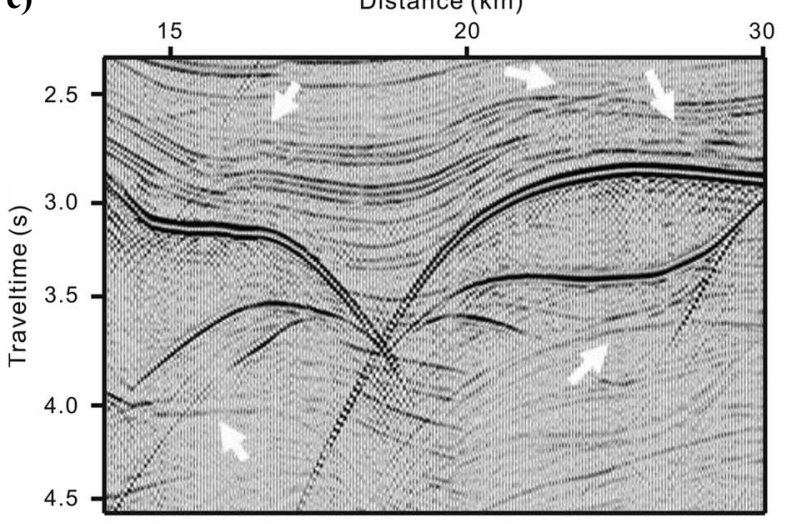

d)

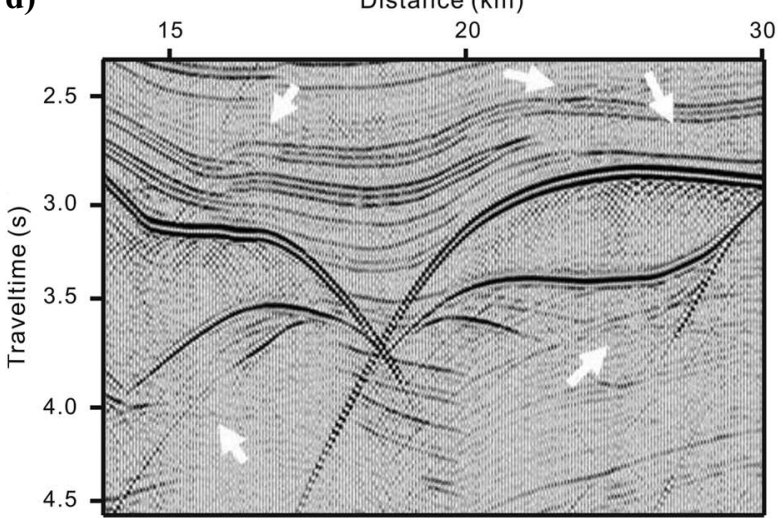

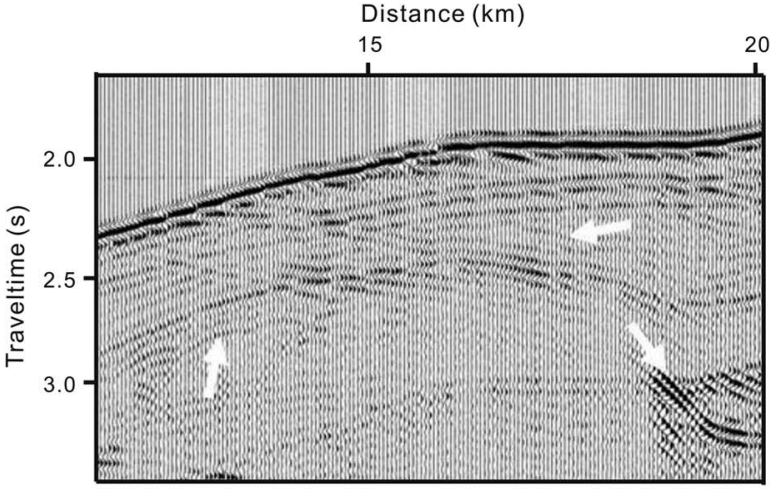
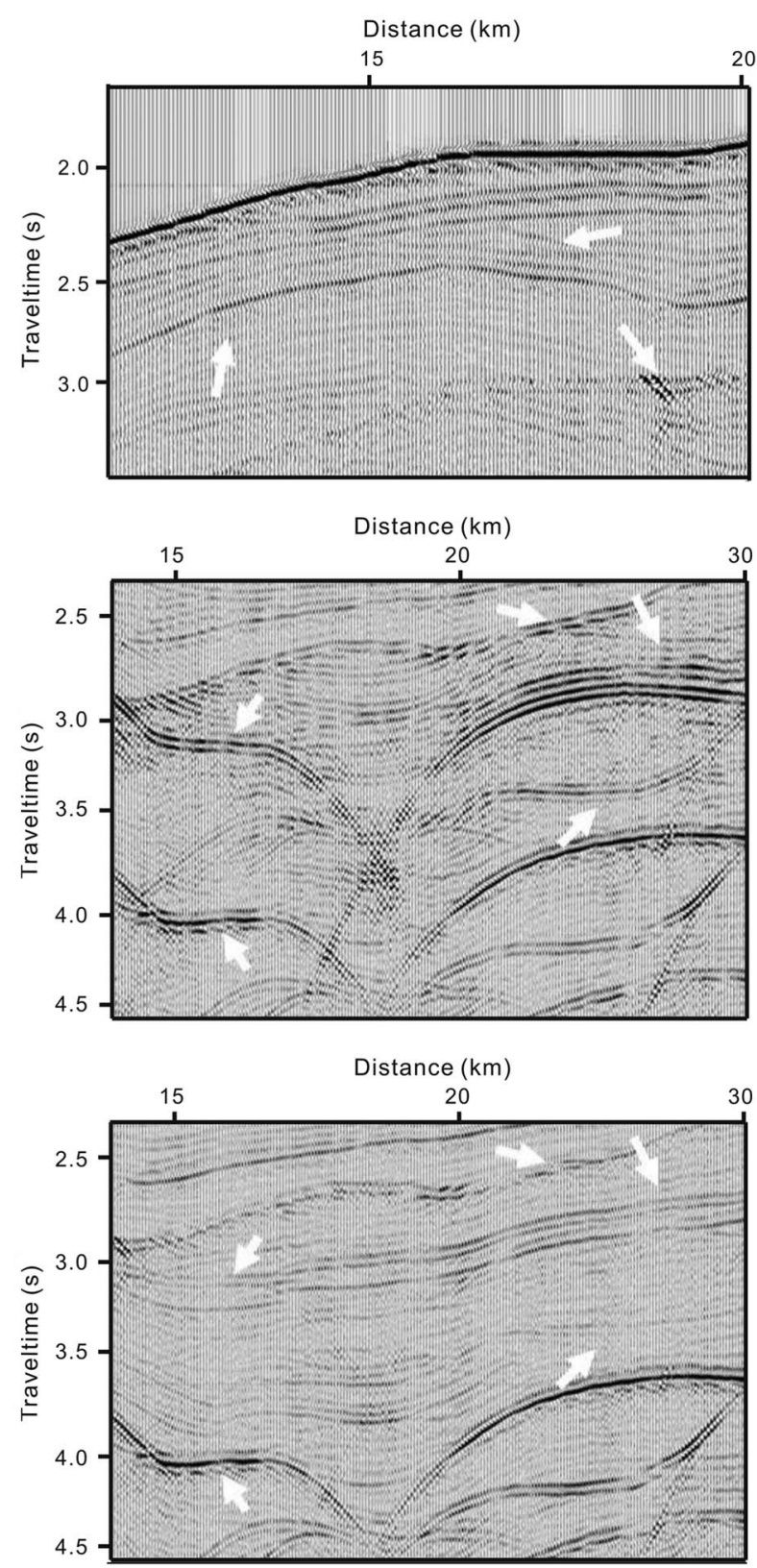

Figure 4. Partial zoom-in of Figure 3: Attenuation result (left) and attenuated energy (right). (a), (c) The result of the conventional short-window (800 ms) adaptive subtraction; (b), (d) the result of adopting the masking filter and subtraction according to orders. 
a)

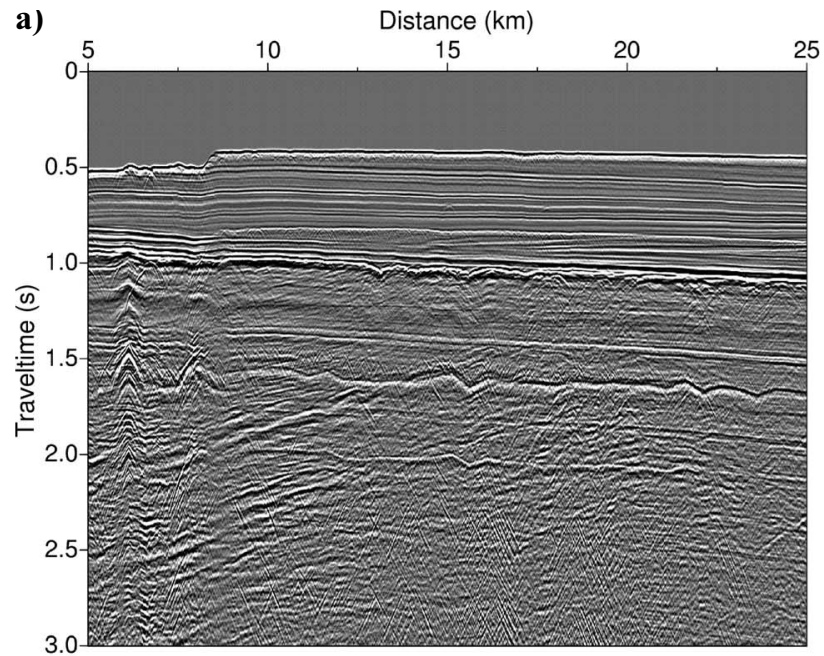

b)

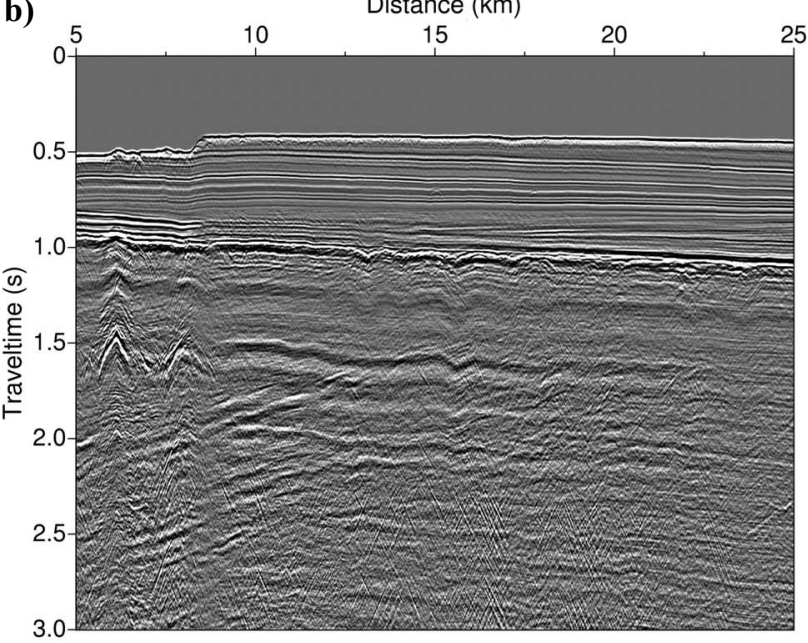

a)

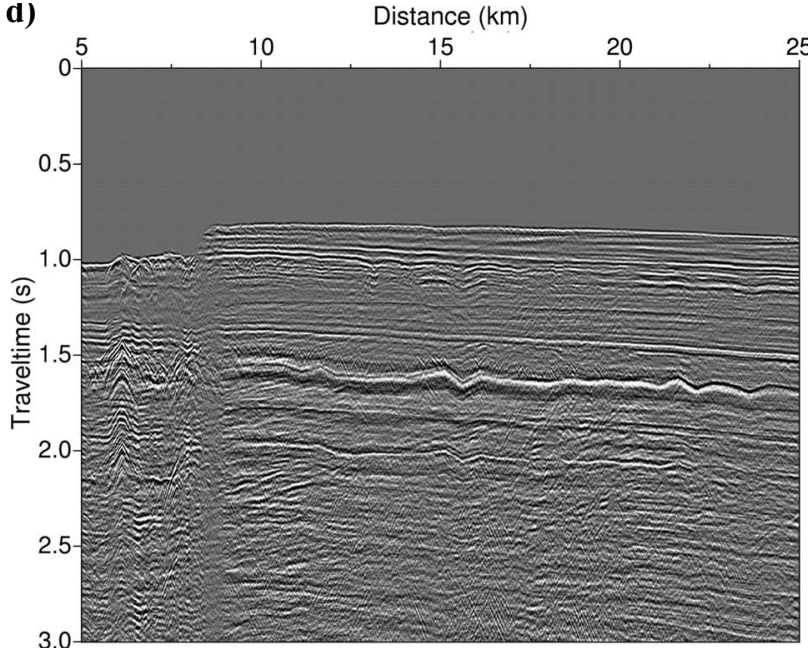

e)

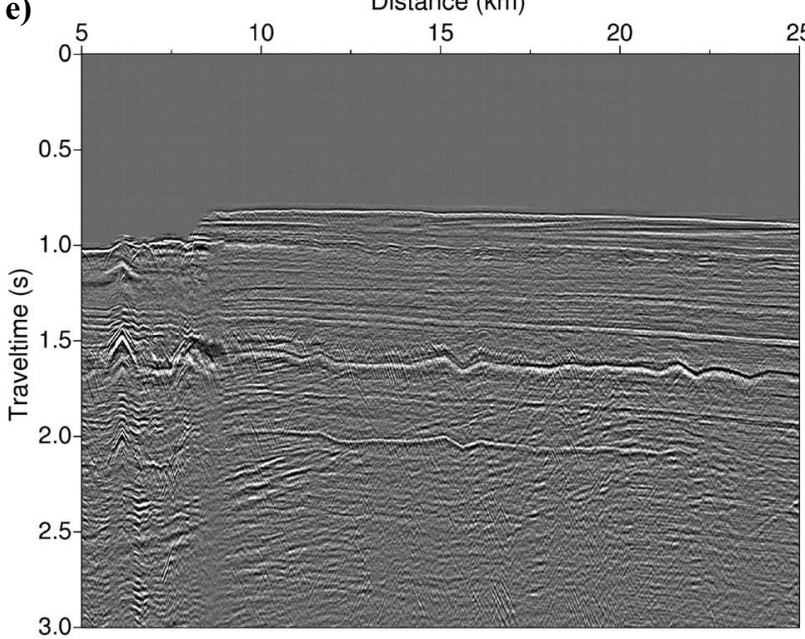

c)

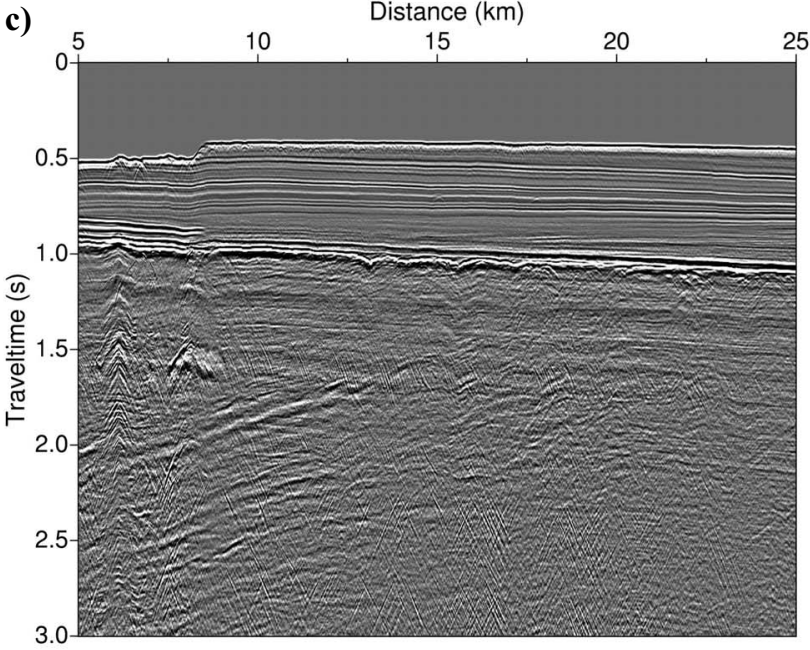

${ }^{28}$

.

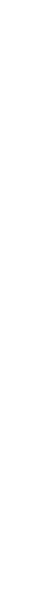

Figure 5. A multiple attenuation example of real marine seismic data. (a) Stack profile of raw data. Multiple attenuation result by (b) using conventional adaptive subtraction with masking filter, and (c) using subtraction according to orders and the iterative EMCM filtering with masking filter. (d) The difference between ( $a$ and b), and (e) the difference between ( $a$ and c).

adaptive subtraction. In addition, some primaries are partially attenuated around the area by mistake, and some high-order multiples (located at $40-70 \mathrm{~km}, 1.6 \mathrm{~s}$ and $2.0 \mathrm{~s}$ ) have been oversubtracted.
Figure $5 \mathrm{c}$ is the multiple attenuation result with the masking and subtracting in orders. The iterative EMCM filter is used in subtraction. Figure $5 \mathrm{~d}$ and e shows the multiple energy attenuated from Fig- 
ure $5 \mathrm{~b}$ (difference of Figure 5a and b) and Figure 5c, respectively. The new method has removed the aliases below the unconformable layers and preserved the primary energy well while removing more multiples.

\section{CONCLUSIONS}

We successfully improved multiple attenuation through the following three schemes.

1) Iterative EMCM filtering: The EMCM filter enables a long window with a short-length filter to give a satisfactory result for most cases. Its iterative application could improve the multiple attenuation result.

2) Multiple subtraction with a masking filter: The masking filter can preserve most of the primary energy from the raw data before subtraction. The multiple subtraction then is performed on the remaining part of the data. As a result, multiples can be attenuated fully and the primaries preserved better.

3) Multiple attenuation according to orders: Different-order multiples have different properties because of the absorption rate and reflectivity. We suggest subtracting them separately according to their orders to obtain a more precise matching filter. Consequently, the result of multiple attenuation could be improved.

\section{ACKNOWLEDGMENTS}

We are grateful to the sponsors of the Centre for Reservoir Geophysics, Imperial College London, for supporting this research. We also thank two anonymous reviewers and the editors for their helpful comments about earlier drafts.

\section{REFERENCES}

Baumstein, A., and M. T. Hadidi, 2006, 3D surface-related multiple elimina- tion: Data reconstruction and application to field data: Geophysics, 71, no. 3, E25-E33.

Berkhout, A. J., and D. J. Verschuur, 1997, Estimation of multiple scattering by iterative inversion: Part I — Theory: Geophysics, 62, 1586-1595.

Guitton, A., 2005, Multiple attenuation in complex geology with a patternbased approach: Geophysics, 70, no. 4, V97-V107.

Hugonnet, P., 2002, Partial surface related multiple elimination: 72nd Annual International Meeting, SEG, Expanded Abstracts, 2102-2105.

Kaplan, S. T., and K. A. Innanen, 2008, Adaptive separation of free-surface multiples through independent component analysis: Geophysics, 73, no. 3, V29-V36.

Kelamis, P. G., D. J. Verschuur, K. E. Erickson, C. L. Robert, and R. M. Burnstad, 2002, Data-driven internal multiple attenuation - Applications and issues on land data: 72nd Annual International Meeting, SEG, Expanded Abstracts, 2035-2038.

Landa, E., I. Belfer, and S. Keydar, 1999, Multiple attenuation in the parabolic t-p domain using wavefront characteristics of multiple generating primaries: Geophysics, 64, 1806-1815.

Lu, W. K., 2006, Adaptive subtraction using independent component analysis: Geophysics, 71, no. 5, S179-S184.

Spitz, S., 1999, Pattern recognition, spatial predictability, and subtraction of multiple events: The Leading Edge, 18, 55-58.

Treitel, S., 1970, Principles of digital multichannel filtering: Geophysics, 35 , 785-811.

Verschuur, D. J., and A. J. Berkhout, 1997, Estimation of multiple scattering by iterative inversion: Part II - Practical aspects and examples: Geophysics, 62, 1596-1611.

Verschuur, D. J., A. J. Berkhout, and C. P. A. Wapenaar, 1992, Adaptive surface-related multiple elimination: Geophysics, 57, 1166-1177.

Wang, Y., 2003a, Multiple attenuation, coping with the spatial truncation effect in the Radon transform domain: Geophysical Prospecting, 51, 75-87. , 2003b, Multiple subtraction using an expanded multichannel matching filter: Geophysics, $\mathbf{6 8}, 346-354$.

, 2004, Multiple prediction through inversion: A fully data-driven concept for surface-related multiple attenuation: Geophysics, $\mathbf{6 9}, 547-553$.

, 2007, Multiple prediction through inversion: Theoretical advancements and real data application: Geophysics, 72, no. 2, V33-V39.

Weglein, A. B., F. A. Gasparotto, P. M. Carvalho, and R. H. Stolt, 1997, An inverse-scattering series method for attenuating multiples in seismic reflection data: Geophysics, 62, 1975-1989.

Wiggins, J. W., 1988, Attenuation of complex water-bottom multiples by wave-equation-based prediction and subtraction: Geophysics, 53, $1527-1539$

Zhou, B., and S. A. Greenhalgh, 1994, Wave-equation extrapolation-based multiple attenuation: 2-D filtering in the f-k domain: Geophysics, 59, 1377-1391.

, 1996, Multiple suppression by 2D filtering in the parabolic tau-p domain: a wave-equation-based method: Geophysical Prospecting, 44, 375-401. 